\title{
Stability of Chaotic and Hyperchaotic Finance Systems
}

\section{Ahmmed Mohammed Juma'a \\ Department of Mathematics \\ College of Computer Sciences and \\ Mathematical \\ Mosul University}

$15 / 02 / 2012$

\author{
Ala'a Abdul-Raheem Hammodat \\ Department of Mathematics \\ College of Education \\ Mosul University
}

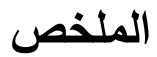

في هذا البحث قدمنا دراسـة لنظام مضطرب (كثير الاضطراب) مستقل ذاتي ثلاثي

(رباعي) الأبعاد هو نظام فينانس، وحصلنا على استقرارية لهذا النظام، إذ تبين بـان النظام النطام

المضطرب (الثلاثي) يكون مستقر عند نقطنيه الحرجتين عندما يكون قيمة المتغير 1 >

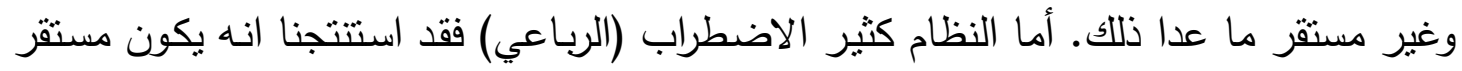

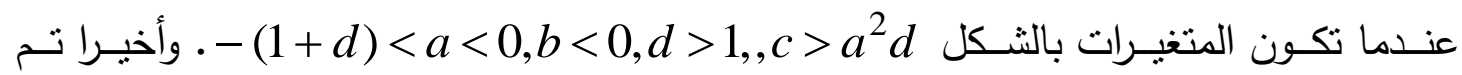

إعطاء أمثلة نوضيحية لكلا النظامين.
\end{abstract}

\begin{abstract}
In this paper, a three (four) dimensional autonomous Finance chaotic (hyperchaotic) system is considered, and we obtained the stability of this systems. We show that the chaotic (three dimensional) system is asymptotically stable at the critical points when $0<a<1$, and unstable otherways. While the hyperchaotic (four dimensional) system is asymptotically stable if the variables has the form $-(1+d)<a<0, b<0, d>1, c>a^{2} d$. Finally, illustrative examples are given.
\end{abstract}

Keywords: Finance chaotic and hyperchaotic systems, stability, Routh-Hurwitz

\section{Introduction:}

Over the past decades, chaos has been found to be very useful and has great potential in many engineering-oriented applied fields such as in encryption and communications, power systems protection, liquid mixing, information sciences, and so on $[3,13]$. 
In 1963, Lorenz discovered the first classical chaotic system[2]. In 1976, O.E. Rössler constructed a several three-dimensional quadratic autonomous chaotic system[10]. In 1999, Chen and Ueta found the dual system of Lorenz system via chaotification approach, in the sense of Lorenz system with $a_{12} a_{21}>0$ and Chen system with $a_{12} a_{21}<0$ from the definition of Vanecek and Celikovsky [3], where $a_{12}$ and $a_{21}$ are the corresponding elements in the linear part matrix $A=\left(a_{i j}\right)_{3 \times 3}$ of the system. In 2002, L $\ddot{u}$ and Chen discovered the critical chaotic system between the Lorenz and Chen system[5], satisfying $a_{12} a_{21}=0$, and the same year, $L \ddot{u}$ et al. unified the above three chaotic systems into a new chaotic system - unified chaotic system [6].

Hyperchaotic system is usually defined as a chaotic system with more than one positive Lyapunov exponent [14]. As we know now, there are many hyperchaotic system discovered in the high dimension social and economical systems $[1,10,12,15]$. Typical examples are fourdimensional hyperchaotic Rössler system [10], four dimensional LorenzHaken system[1], four-dimensional hyperchaotic Chua's circuit [12], and four-dimensional hyperchaotic Chen system[15]. Since hyperchaotic system has the characteristics of high capacity, high security and high efficiency, it has broadly applied potential in the nonlinear circuits, secure communications, lasers, neural networks, biological systems, and so on.

\section{Dynamical Behavior of The Finance Chaotic (Hyperchaotic) Attractors:}

Recent works [7-9] have reported a dynamic model of finance, composed of three first-order differential equations. The model describes the time variations of three state variable: the interest rate $\mathrm{x}$, the investment demand $\mathrm{y}$ and the price index $\mathrm{z}$, By choosing an appropriate coordinate system and setting appropriate dimensions for each state variable, references [7-9] offer the simplified finance system as:

$$
\begin{aligned}
& \dot{x}=-a(x+y) \\
& \dot{y}=-y-a x z \\
& \dot{z}=b+a x y
\end{aligned}
$$

where $(x, y, z)^{T} \in R^{3}$ is the state variables of the system, $a, b$ are parameters and $b>a$.

In light of the thought of G.Chen [15], we construct a hyperchaotic finance system by introducing a state feedback controller $\mathrm{w}$ to the system (1). The new controlled system has the form of:

$$
\begin{aligned}
& \dot{x}=-a(x+y)+w \\
& \dot{y}=-y-a x z \\
& \dot{z}=b+a x y \\
& \dot{w}=-c x z-d w
\end{aligned}
$$


Where $(x, y, z, w)^{T} \in R^{4}$ and $a, b$ are the parameters of the $\operatorname{system}(2)$, and $c$ is constant[4].

In the following briefly describe some basic properties of the systems (1) and (2) respectively.

\section{Symmetry and Invariance:}

First, note that the system (1) and (2) has a semmetry $S$ respectively, because the transformations:

$$
\begin{aligned}
& S:(x,-y, z) \rightarrow(-x, y, z) \\
& S:(x,-y, z, w) \rightarrow(-x, y, z,-w)
\end{aligned}
$$

Which permits systems invariants for all values of the system parameters $a, b, \mathrm{c}, \mathrm{d}$. Obviously, the $\mathrm{z}-$ axis itself is an orbit, that is if $x=0, y=0$, $(x=0, y=0, w=0)$ at $t=0$ then $x=0, y=0 \quad(x=0, y=0, w=0)$ for all $t>0$ respectively. Moreover, the orbit on $z$-axis tend to the origin as $t \rightarrow \infty$. And the transformation $S$ indicates that the systems (1) and (2) are symmetrical on the $z$-axis, for instance, if $\varphi$ is the solution of the systems (1) and (2), and $S \varphi$ is too.[4]

\section{Dissipative:}

The systems (1) and (2) can be dissipative system, because the divergence of the vector field, also called the trace of the Jacobian matrix is negative if and only if $a>-1,(a+d>-1)$.

$$
\begin{aligned}
& \operatorname{div} \vec{V}=\frac{\partial \dot{x}}{\partial x}+\frac{\partial \dot{y}}{\partial y}+\frac{\partial \dot{z}}{\partial z}=\operatorname{Tr}(J)=-(a+1) \\
& V(t)=V(0) e^{-(a+1) t} \\
& \operatorname{div} \vec{V}=\frac{\partial \dot{x}}{\partial x}+\frac{\partial \dot{y}}{\partial y}+\frac{\partial \dot{z}}{\partial z}+\frac{\partial \dot{w}}{\partial w}=\operatorname{Tr}(J)=-(a+d+1) \\
& V(t)=V(0) e^{-(a+d+1) t}
\end{aligned}
$$

So, the systems (1) and (2) will always be dissipative if and only if when $a+1>0,(a+d+1>0)$.

with an exponential: $\frac{d \vec{V}}{d t}=e^{-(a+1)},\left(\frac{d \vec{V}}{d t}=e^{-(a+d+1)}\right)$ see [4].

\section{Critical Points:}

The critical points of the systems (1) and (2) can be easily found by solving the three (four) equations $\dot{x}=\dot{y}=\dot{z}=0,(\dot{x}=\dot{y}=\dot{z}=\dot{w}=0)$ respectively, which leads to 


$$
\begin{array}{r}
-a(x+y)=0 \\
-y-a x z=0 \\
b+a x y=0
\end{array}
$$

$$
\begin{aligned}
-a(x+y)+w & =0 \\
-y-a x z & =0 \\
b+a x y & =0 \\
-c x z-d w & =0
\end{aligned}
$$

It can be easily verified that the system (1) has two critical points

$$
P_{+}(\sqrt{b / a},-\sqrt{b / a}, 1 / a), P_{-}(-\sqrt{b / a}, \sqrt{b / a}, 1 / a)
$$

If $b / a>0$, but if $b / a<0$, there is no critical points; as for $b / a=0$, there is only one critical point but which dependent on the value of the parameter $a$, which is denoted as $(0,0,1 / a), a \in R$.

While when the parameters $a, b, c, d$ satisfy $a b d\left(a^{2} d-c\right)>0$, the system (2) has two equilibrium points:

$$
P_{-}\left(-\frac{b}{a A}, A, \frac{A^{2}}{b}, \frac{c A}{a d}\right), P_{+}\left(\frac{b}{a A}, A, \frac{A^{2}}{b},-\frac{c A}{a d}\right)
$$

Where $A=\sqrt{\frac{a b d}{a^{2} d-c}}$. Obviously, $P_{-}$, and,$P_{+}$are symmetric about $x, y$-axis $(x, y, w$-axis $)$ for any parameters $a, b, c, d .[4]$

\section{Helping Results:}

\section{Remark 1 (Routh-Hurwitz Test) [11]:}

All roots of the indicated polynomial have negative real parts precisely when the given conditions are met:

$$
\begin{aligned}
& \lambda^{3}+a \lambda^{2}+b \lambda+c: a>0, c>0, a b-c>0 \\
& \lambda^{4}+a \lambda^{3}+b \lambda^{2}+c \lambda+d: a>0,(a b-c)>0,(a b-c) c-a^{2} d>0, d>0
\end{aligned}
$$

\section{Remark 2 (Critical Cases) [11]:}

Critical cases in the theory of stability for differential equation means, that cases when the real part of all roots of the characteristic equation have no positive with the real part of at least one root being zero, other express which is neither stable nor unstable.

Now, we only needs to consider the stability of system (1) and (2) at the critical points $P_{+}(\sqrt{b / a},-\sqrt{b / a}, 1 / a)$ and $P_{+}\left(\frac{b}{a A}, A, \frac{A^{2}}{b},-\frac{c A}{a d}\right)$ respectively. Because the system (1) is invariant under the transformation $(x,-y, z) \rightarrow(-x, y, z)$

Under the linear transformation $(x, y, z) \rightarrow(X, Y, Z)$

$$
T=\begin{aligned}
& x=X+\sqrt{b / a} \\
& y=y-\sqrt{b / a} \\
& z=Z+1 / a
\end{aligned}
$$


After substitute (9) in (1) we get:

$$
\begin{aligned}
& \dot{X}=-a(X+Y) \\
& \dot{Y}=-X-Y-a X Z-a \sqrt{b / a} Z \\
& \dot{Z}=a \sqrt{b / a}(Y-X)+a X Y
\end{aligned}
$$

Also, since the system (2) is invariant under the transformation $(x,-y, z,-w) \rightarrow(-x, y, z, w)$

Under the linear transformation $(x, y, z, w) \rightarrow(X, Y, Z, W)$

$$
T=\begin{gathered}
x=X-\frac{b}{a A} \\
y=Y+A \\
z=Z+\frac{A^{2}}{b} \\
w=W+\frac{c A}{a d}
\end{gathered}
$$

After substitute (11) in (2) we get:

$$
\begin{aligned}
& \dot{X}=-a(X+Y)+W \\
& \dot{Y}=-\frac{a A^{2}}{b} X-Y+\frac{b}{A} Z-a x Z \\
& \dot{Z}=a A X-\frac{b}{A} Y+a X Y \\
& \dot{W}=-\frac{c A^{2}}{b} X+\frac{b c}{a A} Z-d W-c X Z
\end{aligned}
$$

\section{Remark 3[4]:}

The characteristic polynomial of system (12) at the critical $P_{+}$is:

$$
\begin{aligned}
& f(\lambda)=\lambda^{4}+(1+a+d) \lambda^{3}+\left(a+d+a d+\frac{a b c^{2} d+a d-a^{3} b d}{b\left(a^{2} d-c\right)}\right) \lambda^{2}+\ldots \\
& \ldots \ldots+\left(2 a^{2} b-b c+a b d-\frac{a b c+b d c}{a d}\right) \lambda+2\left(a^{2} b d-b c\right)=0
\end{aligned}
$$

In [4], studied the stability and control of system (2) when the parameters $a=3, b=15, c=0.2, d=0.12$, and we found that the some eigenvalues of the characteristic polynomial (remark 3 ) has positive real parts $(0.708300,0.032247,0$ and -4.713900$)$. Thus the equilibrium point $P_{+}$is unstable. Similarly, the equilibrium point $P_{-}$is unstable. In this paper a new chaotic (hyperchaotic) finance system is constructed, and stabilization of the chaotic (hyperchaotic) finance system is achieved. when $a, b,(a, b, c, d)$ are parameters. 


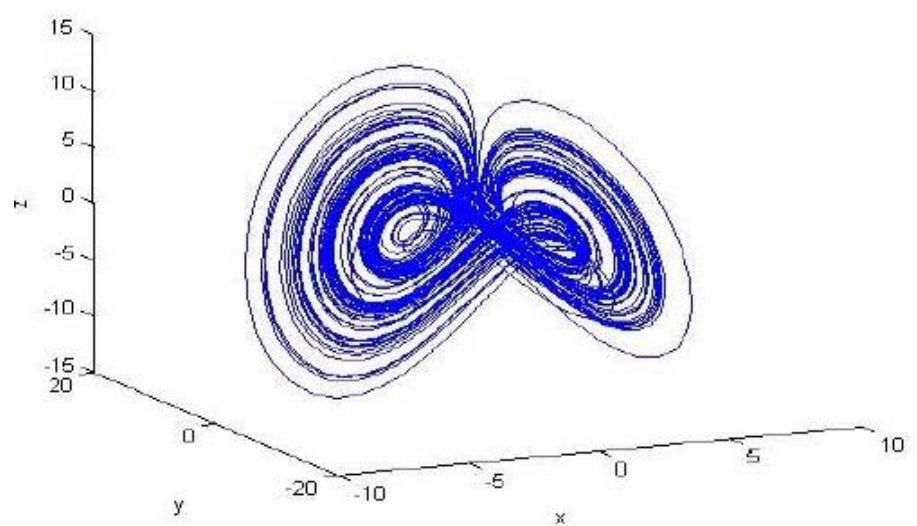

Strange attractor of fnance system(1) when $a=3, b=15$

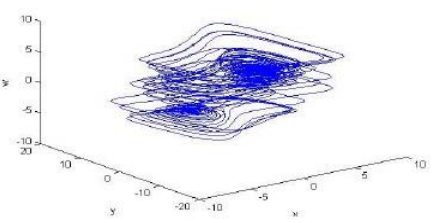

(a) $3 \mathrm{D}$ view in the $x-y-w$ space

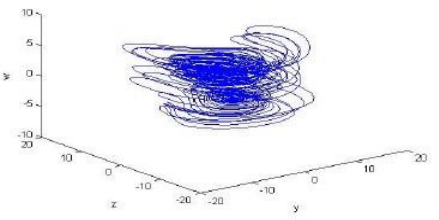

(c) 3D view in the $y-z-w$ space.

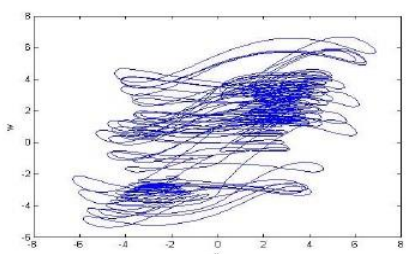

(e) Projection on the $x-w$ plane.

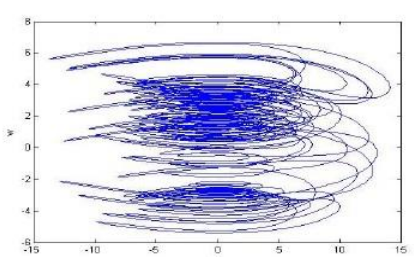

(g) Projection on the $z-w$ plane.

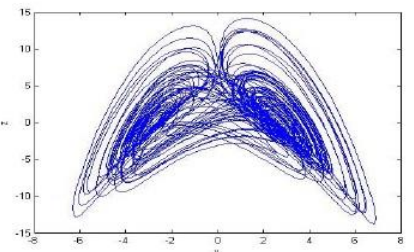

(i) Projection on the $x-z$ plane.

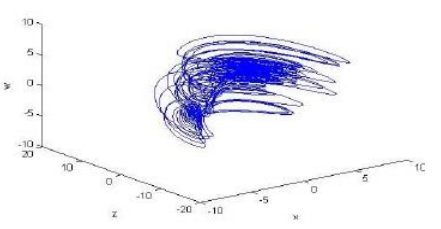

(b) 3D view in the $x-z-w$ space.

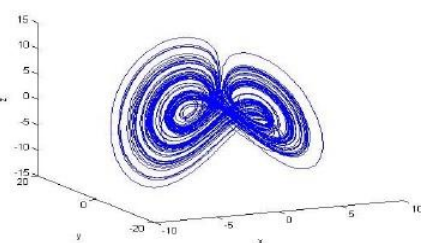

(d) 3D view in the $x-y-z$ space.

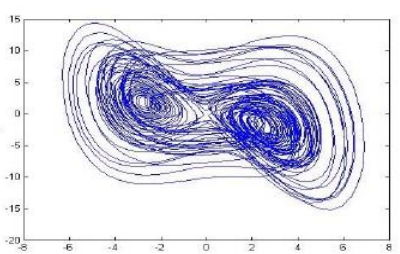

(f) Projection on the $x-y$ plane.

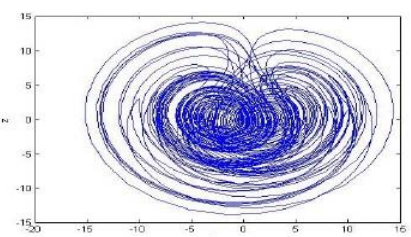

(h) Projection on the $y-z$ plane

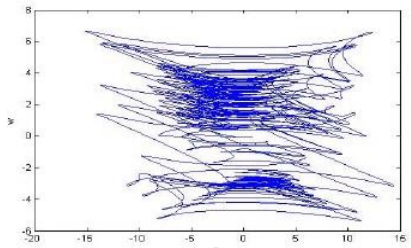

(j) Projection on the $y-w$ plane.

Phase portraits of hyperchaotic finance system(2) when $a=3, b=15, c=0.2, d=0.12$ 


\section{Main result:}

Theorem1: In system (10),

1- if $a \in(0,1)$, and $b>0$, the critical point $P_{0}^{\prime}(0,0,0)$ is asymptotically stable.

2- if $a \in(1, \infty)$, then the critical point $P_{0}^{\prime}(0,0,0)$ is unstable.

3 - if $a=1$, then system (10) is critical case.

Proof: At the critical point $P_{0}^{\prime}(0,0,0)$, system (10) is linearized, the Jacobian matrix is defined as:

$$
J_{+}=\left[\begin{array}{ccc}
-a & -a & 0 \\
-1 & -1 & -a \sqrt{b / a} \\
-a \sqrt{b / a} & a \sqrt{b / a} & 0
\end{array}\right]_{(0,0,0)}
$$

and the characteristic polynomial is:

$$
f(\lambda)=\lambda^{3}+(a+1) \lambda^{2}+a b \lambda+2 a^{2} b=0
$$

Obviously, the two points $P_{\mp}$ have the same stability. Let $A=a+1, B=a b$, and,$C=2 a^{2} b$, then the Routh- Hurwitz condition (remark 2) lead to the conclusion that the real part of the roots $\lambda$ are negative if and only if $A>0, B>0$, and,$A B-C>0$. Since $A=a+1$ and $a$ is positive parameter. Consequently $A>0$ always, $C>0$ when $a, b>0$ and $A B-C>0$ when $a \in(0,1)$ and $b>0$, and hence when $a \in(0,1)$, the system(10) is asymptotically stable, the proof of first condition is complete. While when $a \in(1, \infty)$, then the system (10) is unstable. Finally, if $a=1$, then system (10) has critical case.

Theorem2: The solution of system (12) at the critical point $P_{0}^{\prime}(0,0,0)$ is:

1 - asymptotically stable if $-(1+d)<a<0, b<0, d>1$, and, $c>a^{2} d$.

2- unstable if $-(1+d)<a<0, b<0, d>1$, and, $c<a^{2} d$.

3- critical case if $-(1+d)<a<0, b<0, d>1$, and, $c=a^{2} d$.

Proof: Using Routh -Hurwitz criterion, the equation (13), has all roots with negative real parts if and only if the conditions are satisfied as follows:

$$
\begin{aligned}
& A>0, \\
& D>0, \\
& A B-C>0 \\
& (A B-C) C-A^{2} D>0,
\end{aligned}
$$

Since $A=(a+d+1), \quad B=\left(a+d+a d+\frac{a b c^{2} d+a d-a^{3} b d}{b\left(a^{2} d-c\right)}\right)$ $C=\left(2 a^{2} b-b c+a b d-\frac{a b c+b d c}{a d}\right), a n d, D=2\left(a^{2} b d-b c\right)$. Consequently, after applying the conditions $-(1+d)<a<0, b<0, d>1$, and, $c>a^{2} d$, we get 
that the system (12) is asymptotically stable always, and if $-(1+d)<a<0, b<0, d>1$, and, $c<a^{2} d$, we get that the system (12) is unstable. Finally if $-(1+d)<a<0, b<0, d>1$, and, $c=a^{2} d$, then satisfied remark 2 , hence the system (12) is critical case, the proof is complete.

\section{Illustrative Examples:}

Example 1: Investigate for stability of system (1) at the critical point $P_{0}^{\prime}(0,0,0)$, Where $a=\frac{1}{2}, b=2$

Solution: from equation (15) we get

$$
\begin{aligned}
& f(\lambda)=\lambda^{3}+(a+1) \lambda^{2}+a b \lambda+2 a^{2} b=0 \\
& \Rightarrow f(\lambda)=\lambda^{3}+\frac{3}{2} \lambda^{2}+\lambda+1=0
\end{aligned}
$$

And by using Routh - Hurwitz conditions we get $a_{1}=\frac{3}{2}>0, a_{3}=1>0$, and,$a_{1} a_{2}-a_{3}=\frac{1}{2}>0$, hence the system is asymptotically stable and the first part of theorem (1) is satisfied. While if $a=2, b=4$, we get $a_{1}=3>0, a_{3}=32>0$, and, $a_{1} a_{2}-a_{3}=-8<0$ and the system is unstable. And if $a=1, b=2$, then $a_{1}=2>0, a_{3}=4>0$, and,$a_{1} a_{2}-a_{3}=0$ and the system (10) is critical case.

Example 2: Investigate for stability of system (12) at critical point $P_{0}^{\prime}(0,0,0,0)$, when $a=-3, b=-2, d=3, c>27$, let,$c=28$

Solution: from equation (13) we get:

$$
f(\lambda)=\lambda^{4}+(1+a+d) \lambda^{3}+\left(a+d+a d+\frac{a b c^{2} d+a d-a^{3} b d}{b\left(a^{2} d-c\right)}\right) \lambda^{2}+\ldots
$$

$\ldots \ldots+\left(2 a^{2} b-b c+a b d-\frac{a b c+b d c}{a d}\right) \lambda+2\left(a^{2} b d-b c\right)=0$

$\Rightarrow f(\lambda)=\lambda^{4}+\lambda^{3}+(6961.5) \lambda^{2}+38 \lambda+4=0$, and by using Routh-Hurwitz conditions we get,

$$
a_{1}=1>0, a_{4}=4>0, a_{1} a_{2}-a_{3}=6923.5>0, \text { and },\left(a_{1} a_{2}-a_{3}\right) a_{3}-a_{1}^{2} a_{4}=263089>0
$$

Hence, the system(12) is asymptotically stable.

While if $c=26, \Rightarrow a_{4}<0$, and the system (12) is unstable.

Finally, if $c=27, \Rightarrow a_{4}=0$, and the system (12) is critical case.

Example 3: Investigate for stability of system (12) at critical point $P_{0}^{\prime}(0,0,0,0)$, when $b=-2, d=1.1, a>-2.1$, let $, a=-2, c>4.4$, let,$c=5$

Solution: from equation (13) we get

$$
\begin{aligned}
& f(\lambda)=\lambda^{4}+(1+a+d) \lambda^{3}+\left(a+d+a d+\frac{a b c^{2} d+a d-a^{3} b d}{b\left(a^{2} d-c\right)}\right) \lambda^{2}+\ldots \\
& \ldots \ldots+\left(2 a^{2} b-b c+a b d-\frac{a b c+b d c}{a d}\right) \lambda+2\left(a^{2} b d-b c\right)=0
\end{aligned}
$$


and by using Routh-Hurwitz conditions we get, $a_{1}=0.1>0, a_{4}=2.4>0, a_{1} a_{2}-a_{3}=4.715757576>0$, and,$\left(a_{1} a_{2}-a_{3}\right) a_{3}-a_{1}^{2} a_{4}>0$ Hence, the system(12) is asymptotically stable.

While if $c=4, \Rightarrow a_{4}<0$, and the system(12) is unstable.

Finally, if $c=4.4, \Rightarrow a_{4}=0$, and the system(12) is critical case.

\section{Conclusions:}

In this paper, a three (four) dimensional autonomous Finance chaotic (hyperchaotic) systems are studied, there are obtained stability of these systems. We show that the three dimensional system is asymptotically stable at the critical points when $0<a<1$, and unstable in others. While the four dimensional hyperchaotic system is asymptotically stable if the variables has the form $-(1+d)<a<0, b<0, d>1, c>a^{2} d$.

\section{References}

1) C.Z.Ning, H.Haken, "Detuned lasers and the complex Lorenz Equations", subcritical and super-critical Hopf bifurcations. phys. Rev. A.(41), pp.3826-3837,(1990).

2) E.N. Lorenz "Deterministic nonperiodic flow" [J]. Journal of the Atmospheric Science, Vol. (20), 2,PP. 130-141, (1963)

3) G.Chen, J.Lü, "Dynamic of the Lorenz System Family" Analysis, Control and Synchronization, Science Press, Beijing, (2003) (in Chinese).

4) J. Ding, W. Yang, Hongxing Yao, "A New Modified Hyperchaotic Finance System and its Control", International journal of nonlinear scienc, Vol. (8),pp.59-66, (2009).

5) J.Lü, G. R. Chen "A new chaotic attractor coined" [J]. Int, J. of Bifurcation and Chaos, Vol.(12), 3,P.P.659-661, (2002)

6) J.Lü "Bridge the gap between the Lorenz system and the Chen system" [J]. Int.J. of Bifurcation and Chaos, Vol. (12),12,PP. 29172926, (2002).

7) J.H.Ma, Y.S.Chen. "Syudy for the bifurcation topological structure and the global complicated character of a kind of nonlinear finance system"(I). Appl.Mech (Englished), Vol.(22), pp.1240-1251, (2001). 
8) J.H.Ma, Y.S.Chen. "Syudy for the bifurcation topological structure and the global complicated character of a kind of nonlinear finance system"(II). Appl.Mech (Englished), Vol.(22), pp.1375-1382, (2001).

9) J.H.Ma, R.B,Y.S. Chen. "Implusive control of chaotic Attractors in nonlinear chaotic systems". Appl. Mech., Vol.(25), pp.1889-894, (2004).

10) O.E.Rossler. "An equation for hyperchaos", Phys.Lett.A, 71(2-3), pp.155- 157, (1979).

11) R.L. Borrelli, C.S. Coleman "Differential Equations" New-York, John Wiley and Sons, Inc, (1998).

12) T.Kapitaniak, L.O.Chua. "Hyperchaos attractor of unidirectionally coupled Chua's circuit", Int.J. Bifurcat. Chaos, Vol.(4),2, pp.477482, (1994).

13) Y. Mao, G.Chen "Chaos-Based Image Encryption" in: E. Bayro(Ed.), Handbook of Computational Geometry, Springer, Berlin, (2003).

14) Y.Takahashi, H.Nakano, T.Saito. "A simple hyperchaos generator based on impulsive switching", IEEE. Trans. Circuits. syst.II.51, pp.468-472, (2004).

15) Y.X.Li, W.K.S. Tang, G. Chen. "Generating hyperchaos via state feedback control" Int. J. Bifurcat. chaos., Vol.(15), 10, pp.33673375 , (2005). 